\title{
DIVISION VIII / WORKING GROUP SUPERNOVA
}

\author{
CO-CHAIRS \\ MEMBERS
}

\author{
Wolfgang Hillebrandt and Brian P. Schmidt \\ Edward Baron, Stefano Benetti, Sergey I. Blinnikov, \\ Sergey I. Blinnikov, David R. Branch, \\ Enrico Cappellaro, Alexei V. Filippenko, \\ Claes Fransson, Peter M. Garnavich, \\ Daniel W. E. Green, Ariel M. Goobar, Mario Hamuy, \\ Peter H. Hauschildt, Robert P. Kirshner, \\ Bruno Leibundgut, Daniel J. Lennon, Eric J. Lentz, \\ Peter Lundqvist, Robert Mc Graw, Paolo A. Mazzali, \\ W. Peter S. Meikle, Anthony Mezzacappa, \\ Jens C. Niemeyer, Ken'ichi Nomoto, Reynald Pain, \\ Nino Panagia, Ferdinando Patat, Mark M. Phillips, \\ Elena Pian, Guiliano Pignata, Philipp Podsiadlowski, \\ María Pilar Ruiz-Lapuente, Elaine M. Sadler, \\ Brian P. Schmidt, Peter O. Shull, Jason Spyromilio, \\ Nicholas B. Suntzeff, Friedrich-Karl Thielemann, \\ Christopher Tout, Virginia L. Trimble, \\ James W. Truran, Dmitry Yu. Tsvetkov, \\ Massimo Turatto, Massimo della Valle, \\ Schuyler Van Dyk, Wolfgang H. Voges, \\ Nicholas A. Walton, Lifan Wang, J. Craig Wheeler, \\ Kurt W. Weiler, Patricia A. Whitelock, \\ Stanford E. Woosley, Hitoshi Yamaoka, Gang Zhao
}

TRIENNIAL REPORT 2006 - 2009

\section{Introduction}

The Supernova Working Group was re-established at the IAU XXV General Assembly in Sydney, 21 July 2003, sponsored by Commissions 28 (Galaxies) and 47 (Cosmology). Here we report on some of its activities since 2005.

A first WG on supernovae was founded by Fritz Zwicky to coordinate and provide uniform standards for photographic supernova searches in the era of the Palomar $48^{\prime \prime}$ and other Schmidt telescopes. It died with him in 1974. Commission 28 (Galaxies) reestablished a WG-SN (with Virginia Trimble as chair) at the 1982 Patras IAU General Assembly, largely because of concerns that the few events then being discovered (mostly serendipitously) were not receiving enough follow-up observations to be very information. Among other things, the WG had some small part in preparing the astronomical community to take full advantage of SN 1987A. This event made astronomy so supernovaconscious that the WG hardly seemed needed, and it died a natural death at the 1991 IAU General Assembly. 
The Supernova Working Group was re-established at the IAU XXV General Assembly in Sydney, 21 July 2003, sponsored by Commissions 28 (Galaxies) and 47 (Cosmology). Here we report on some of its activities since 2005.

The wish to request permission of the IAU Executive Committee to re-establish a Supernova Working Group arose from the large number of discoveries (up to several 100 per year), some at very large distances, being made by more than half a dozen teams in the past few years. This was beginning to lead to competition for telescope time for follow-up work which would be unnecessary if the scientists involved had a platform for information exchange. LOTOSS was taking up speed. The Nearby Supernova Factory, the Carnegie Supernova Project, ESSENCE, and other projects to discover and observe SNe Ia at low and intermediate redshifts had left the planning stage in Europe, Japan, and the USA and were thought also to benefit from better coordination. Also, special space probes $(S N A P)$ were being discussed aiming at the discovery of hundreds of supernovae at all redshifts alone. Finally, considerable progress in modeling the various types of events made it desirable also to have theoretical results, including explosion models, synthetic light curves, and predicted spectra, easily accessible.

Therefore, the aim of the new WG-SN was $(a)$ to take care of coordinating observational activities, including searches and surveys (e.g., SCP, High- $z$ Team, GOODS, ESSENCE, LOTOSS, CSP, RTN/ESC, SDSS, Pan-STARRS, etc.); (b) to advocate suitable follow-ups; $(c)$ to establish ways to archive SN data and to make them available to the community; $(d)$ to edit an electronic newsletter complementary to the IAU Circulars (the GCN network for $\gamma$-ray bursters being a possible model); and $(e)$ to be the interface with the coming virtual observatory initiatives (AVO, NVO, ASTRO-Grid, GAVO...) for data storage and handling.

\section{Tasks of the Supernova Working Group}

At an inaugural meeting (which was part of the Division VIII/Commission 28 Business Meeting in Sydney) the objectives of the WG were discussed and approved. In particular it was agreed

- to coordinate the nearby searches and the follow-up observations;

- to launch a community effort for a Treasury or Large HST programme to observe type Ia supernovae in the UV;

- to investigate the possibility of having a robotic (2m-class) telescope devoted to supernova observations; and

- to find ways to save supernova data in some form of database, freely accessible to the community.

In addition, it was suggested that the WG should take an active role in the discovery and observation reports of supernovae, presently done via IAU Circulars, which involves some unwanted delay. Here the problem is, however, that rapid communication in an 'unfiltered' way increases the risk of 'false alarms'. A second problem is going to be how to cope with 100's of supernovae per year. A possible solution could be that IAU Circulars could continue to be the place for 'confirmed' discoveries, preceded by rapid communications in a web-based platform.

Finally, Wolfgang Hillebrandt (Garching, Germany) and Brian Schmidt (Mt. Stromlo, Australia) were elected as co-chairs of the new Supernova Working Group. 


\section{Activities of the Supernova Working Group since 2005}

Here we summarize briefly some of the WG-SN activities in the past three years.

\subsection{Coordinated observations of nearby type Ia supernovae}

A new attempt was made to observe a sample of (nearby) type Ia supernovae with HST in the UV in Cycle 17 after the service mission SM4 scheduled for October 2008. Two proposals were submitted with the goal to improve substantially our physical knowledge of SNe Ia and their calibration as cosmological distance probes. One of these proposals was approved (PI: R. Ellis), together with with about 15 other proposals dealing with various aspects of both, thermonuclear and core-collapse supernovae and their progenitors and environment.

A new on-line supernova spectrum archive (SUSPECT) at the University of Oklahoma and is organized and maintained by David Branch and Eddie Baron. It is steadily extending and at present, it contains photometric and/or spectroscopic data of 175 supernovae of various types as well as links to other archives. Similar efforts are underway at LBNL which in the future should include model data also.

\subsection{Conferences and meetings}

In the past years the WG-SN took an active role in organizing several supernova conferences and workshops.

A Joint Discussion on Supernovae: One Millennium after 1006 at the IAU XXVI General Assembly in Prague to celebrate the SN 1006 millennium by reviewing recent progress in understanding supernovae, their remnants, and their application to cosmology. In a stimulating day and a half there were 25 (mostly invited) oral papers, as well as some two dozen posters touching on many observational and theoretical aspects of supernova research. The oral papers focused primarily on type Ia supernovae and their remnants, including observations of SN1006 itself and its more recent cousins, models for SN Ia progenitors, explosion mechanisms, and how they interact with the interstellar and circumstellar medium on the way to becoming remnants, through the application of SN Ia to cosmology.

There was as SNAP workshop at Berkeley in September 2005, with the main emphasis on the use of type Ia supernovae as probes for cosmology. A conference at Cefalu on Sicily in 2006 dealt with the Multicolour Landscape of Compact Objects and their Explosive Origins and another supernova conference at the same site is coming up in the fall of 2008. There was an extended (type Ia) supernova program at the KITP, Santa Barbara, USA, from January through May, 2007, and several conferences and workshops devoted to SN 1987A (20 years after ...).

\section{Future activities: Supernova data bases and related issues}

Setting-up and maintaining supernova data bases will continue to be in the focus of the WG-SN. In the future it will become even more important to combine observational data with detailed model predictions, and virtual observatory initiatives (AVO, NVO, ASTRO-Grid, GAVO ... ) can provide the platform for data storage and handling needed for this purpose. Whether or not the WG-SN can play a significant role in these efforts will have to be seen.

Wolfgang Hillebrandt \& Brian P. Schmidt co-chairs of the Working Group 